\title{
RESTING HEART RATE AND THE RISK OF DEATH AND CARDIOVASCULAR COMPLICATIONS IN PATIENTS WITH TYPE 2 DIABETES MELLITUS
}

\author{
G. S. Hillis ${ }^{1}$, M. Woodward ${ }^{1}$, A. Rodgers ${ }^{1}$, C. Chow ${ }^{1}$, Q. Li ${ }^{1}$, S. Zoungas ${ }^{1}$, A. Patel ${ }^{1}$, R. \\ Webster $^{1}$, G. D. Batty ${ }^{2}$, T. Ninomiya ${ }^{3}$, G. Mancia ${ }^{4}$, N. R. Poulter ${ }^{5}$, and J. Chalmers ${ }^{1}$ \\ ${ }^{1}$ The George Institute for Global Health, Sydney, Australia \\ ${ }^{2}$ Department of Epidemiology and Public Health, University College London, United Kingdom \\ ${ }^{3}$ Faculty of Medical Sciences, Kyushu University, Fukuoka, Japan \\ ${ }^{4}$ Department of Clinical Medicine and Prevention, University of Milano-Bicocca, Italy \\ ${ }^{5}$ Department of Clinical Pharmacology, Imperial College, London, United Kingdom
}

\section{Abstract}

\begin{abstract}
Aim-An association between resting heart rate and mortality has been described in the general population and in patients with cardiovascular disease. There are, however, few data exploring this relationship in patients with type 2 diabetes mellitus. The current study addresses this issue.

Methods-The relationship between baseline resting heart rate and all-cause mortality, cardiovascular death and major cardiovascular events (cardiovascular death, non-fatal myocardial infarction or non-fatal stroke) was examined in 11,140 patients who participated in the Action in Diabetes and Vascular Disease: Preterax and Diamicron Modified Release Controlled Evaluation (ADVANCE) study.
\end{abstract}

Results-A higher resting heart rate was associated with a significantly increased risk of allcause mortality (fully adjusted hazard ratio [HR] 1.15 per $10 \mathrm{bpm}, 95 \%$ confidence interval [CI] $1.08,1.21, \mathrm{p}<0.001$ ), cardiovascular death, and major cardiovascular outcomes without adjustment and after adjusting for age and sex and multiple co-variates. The increased risk associated with a higher baseline resting heart rate is most obvious in patients with previous macrovascular complications (fully adjusted HR for death 1.79 for upper [mean $91 \mathrm{bpm}$ ] $v$ lowest [mean $58 \mathrm{bpm}$ ] fifth of resting heart rate in this sub-group, 95\% CI 1.28,2.50, $\mathrm{p}<0.001$ ).

Conclusions-Among patients with type 2 diabetes, a higher resting heart rate is associated with increased risk of death and cardiovascular complications. It remains unclear whether a higher

\footnotetext{
Address for correspondence: A/Professor Graham Hillis, The George Institute for Global Health, King George V Building, Royal Prince Alfred Hospital, Sydney, NSW 2050, Australia. Tel: +61 28507 2513, Fax: +61 29993 4502, ghillis@ georgeinstitute.org.au. Author contributions: Conception and design of study: GSH, MW, AR, GDB, TN, JC ; Analysis of data: GSH, MW, QL ; Interpretation of data: GSH, MW, AR, CC, QL, SZ, AP, RW, GDB, TN, GM, NRP, JC ; Drafting of article: GSH, MW, JC ; Revision of article: GSH, MW, AR, CC, QL, SZ, AP, RW, GDB, TN, GM, NRP, JC ; Final approval of article: GSH, MW, AR, CC, QL, SZ, AP, RW, GDB, TN, GM, NRP, JC.

Disclosures: Drs Woodward, Zoungas, Patel, Mancia, Poulter and Chalmers have received lecture fees and/or travel expenses from Servier. Drs Woodward, Zoungas, Patel, Poulter and Chalmers have also received grant support from Servier.
} 
heart rate directly mediates the increased risk or is a marker for other factors that determine a poor outcome.

\section{Keywords}

Type 2 diabetes mellitus; heart rate; outcome

Several studies have demonstrated an association between resting heart rate and all cause and cardiovascular mortality.[1,2] This relationship has been described both in the general population and in patients with cardiac disease. In particular, an increased heart rate predicts an unfavourable outcome in patients with heart failure and following an acute coronary syndrome.[1] There are, however, few data specifically exploring the relationship between resting heart rate and cardiovascular outcome in patients with diabetes mellitus. This is surprising given the accessibility of the measure, the strength of the observed relationship in other populations, the high and increasing prevalence of diabetes and the association between heart rate and glucose and insulin levels. In addition, diabetes mellitus is commonly associated with abnormal function of the autonomic nervous system, the main regulator of resting heart rate. The aim of the current study is, therefore, to explore the relationship between resting heart rate at baseline and cardiovascular events and all-cause mortality in a large cohort of patients with type 2 diabetes mellitus.

\section{METHODS}

The Action in Diabetes and Vascular Disease: Preterax and Diamicron Modified Release Controlled Evaluation (ADVANCE) study (ClinicalTrials.gov number NCT00145925) was a factorial trial that recruited 11,140 patients with type 2 diabetes mellitus from 215 centres in 20 countries between June 2001 and March 2003.[3, 4] The study made two randomised comparisons: a double-blind assessment of the efficacy of a fixed combination of perindopril-indapamide ( $2 \mathrm{mg} / 0.625 \mathrm{mg}$ for 3 months, increasing if tolerated to $4 \mathrm{mg} / 1.25 \mathrm{mg}$ thereafter) versus placebo and an open-label evaluation of an intensive glucose lowering regimen based on gliclazide modified release (MR) targeting an $\mathrm{HbAlc}$ of $56.5 \%$, versus standard guideline based glycaemic control. The study was approved by the local ethics committee for each participating centre and all participants provided written informed consent. Detailed study methods[5] and the main results[3, 4] of the ADVANCE study have been previously reported. Briefly, participants in the ADVANCE study were all at least 55 years of age and had been diagnosed with type 2 diabetes after the age of 30 years. In addition, they were required to have had at least one major macro or micro-vascular complication of diabetes or one or more additional cardiovascular risk factor.

Baseline data included estimates of the duration of diabetes, indices of diabetic control and detailed information regarding lifestyle, including self-reported estimates of average weekly exercise. Baseline resting heart rate was measured at the same time as the blood pressure using a digital monitor (Omron HEM-705CP, Omron Healthcare Inc., Lake Forest, Illinois, USA). Recordings were made after the patient had been resting for a minimum of 5 minutes in the seated position. Three readings were taken and the mean of the latter 2 was used. 
Weight, height, urinary albumin creatinine ratio, serum creatinine, fasting lipid levels and haemoglobin A1c (HbA1c), were also measured at baseline.

The current study assesses the relationship between baseline resting heart rate and all-cause mortality, cardiovascular death and major cardiovascular events (a composite of cardiovascular death, non-fatal myocardial infarction or non-fatal stroke; a primary outcome of the ADVANCE study) over the entire length of the trial. All outcomes were validated by an independent adjudication committee who were unaware of treatment allocation.

\section{Statistical analyses}

Baseline resting heart rate was considered both as a continuous variable and after grouping into approximately equal fifths according to its quintiles. Categorical data were summarised using percentages and compared using chi-square tests. Normally distributed continuous data were summarised as mean (standard deviation [SD]) and comparisons made using the $t$ test or a general linear model to determine any difference across fifths). Skewed data are presented as median (inter-quartile range) and compared using the Kruskal-Wallis test.

Cox regression analyses were used to calculate hazard ratios (HR) with $95 \%$ confidence intervals. These were performed without adjustment and in models adjusted for age and sex and for multiple covariates (age, sex, ADVANCE study blood pressure treatment arm, ADVANCE study glycemic control arm, body mass index, duration of diabetes, HbA1c, urinary albumin creatinine ratio, estimated glomerular filtration rate, systolic blood pressure, diastolic blood pressure, history of hospitalisation for heart failure, participation in moderate and/or vigorous exercise for $>15$ minutes at least once weekly, total cholesterol, triacylglycerol level, atrial fibrillation, treatment with calcium channel blockers and treatment with $\beta$-blockers). Subgroup analyses were performed assessing outcome in patients who had and had not experienced a macrovascular complication prior to randomisation in the ADVANCE study, according to treatment with $\beta$-blockers at the time the heart rate was recorded and stratified by the presence or absence of atrial fibrillation (on an electrocardiogram performed at baseline or previously[6]). The linearity of the relationship between resting heart rate and outcome was assessed through likelihood tests by fitting the fifths of resting heart rate as an ordinal variable and residual non-linearity was tested by comparison with fitting the fifths as a categorical variable.[7] Competing risk analyses were performed to determine whether a higher heart rate conferred an increased risk of all-cause mortality, as compared to other outcomes.[8] Analyses were performed using SPSS v18 (SPSS Inc., Chicago, Illinois, USA) and Stata 11 (Statacorp LP, College Station, Texas, USA) and all significance levels are 2-sided.

\section{RESULTS}

Of the 11,140 patients randomised into the ADVANCE study, two individuals did not have their baseline resting heart rate recorded. The remaining 11,138 patients are included in the current analyses. Their mean heart rate was 74 (SD 12) beats per minute (bpm), with a range of 33 to $140 \mathrm{bpm}$. Baseline average resting heart rates are slightly lower in men (73, SD 12, bpm) than women (75, SD 11, bpm, p<0.001) and in older patients (73, SD 12, bpm in 
patients $\ 65$ years old $\mathrm{v} 75, \mathrm{SD} 12, \mathrm{bpm}$ in those $<65$ years of age, $\mathrm{p}<0.001)$. Table 1 shows the relationship between heart rate fifth and a variety of clinical variables.

During a median follow-up of 4.4 years, $879(8 \%)$ patients died, $468(4 \%)$ from cardiovascular causes. One thousand patients (9\%) suffered a major cardiovascular event during follow-up. A higher resting heart rate is associated with a greater hazard of death (allcause and cardiovascular) and major cardiovascular events (figure 1). Adjustment for use of other cardiovascular medications (statins, angiotensin converting enzyme inhibitors or angiotensin receptor blockers), alternate measures of renal function (creatinine or creatinine clearance), lipid fractions (low or high density lipoprotein levels) or other measures of average weekly exercise (participation in vigorous exercise $>15$ minutes per week or number of exercise sessions $>15$ minutes per week) makes minimal difference to the observed hazard associated with a higher heart rate (data not shown). The hazard associated with a faster resting heart rate is also similar in unadjusted and age and sex adjusted models (see supplementary figures 1-3).

The relationship between baseline heart rate and each outcome was linear $(\mathrm{p}<0.001$ for death, $\mathrm{p}=0.003$ for cardiovascular death and $\mathrm{p}=0.05$ for major cardiovascular events) with no evidence of nonlinearity ( $\mathrm{p}=0.14$ for death, $\mathrm{p}=0.96$ for cardiovascular death and $\mathrm{p}=0.48$ for major cardiovascular events). After adjustment for multiple co-variates the hazard of death increases by $15 \%$ (95\% CI $8-21 \%, \mathrm{p}<0.001$ ) for every $10 \mathrm{bpm}$ increase in resting heart rate. Likewise, the hazard of cardiovascular death increases by $16 \%$ (95\% CI 7,25\%, p<0.001) and for all cardiovascular events by $8 \%(95 \% \mathrm{CI} 2,14 \%, \mathrm{p}=0.009)$. This increased hazard is evident in both male and female patients (fully adjusted HR for death 1.11 per $10 \mathrm{bpm}, 95 \%$ CI $1.04,1.19, \mathrm{p}=0.002$ in men and fully adjusted HR for death 1.22 per $10 \mathrm{bpm}, 95 \%$ CI $1.10,1.36, \mathrm{p}<0.001$ in women). In competing risk models (using all-cause mortality as the competing event) the sub-distribution hazard of cardiovascular death increases by $15 \%$ (95\% CI $7,25 \%, \mathrm{p}<0.001)$ and for all major cardiovascular events by $7 \%$ (95\% CI 1,13\%, $\mathrm{p}=0.01)$.

The adverse prognosis associated with a higher heart rate is most evident in diabetic patients who have had a previous macrovascular event (myocardial infarction, stroke, hospital admission for a transient ischemic attack, hospital admission for unstable angina, coronary revascularization, peripheral revascularization or amputation secondary to peripheral vascular disease: figure 2). In this high risk subgroup the fully adjusted hazard ratio for death is 1.79 for patients in the upper fifth of resting heart rate (mean $91 \mathrm{bpm}$ ) when compared to the those in the lowest fifth (mean $57 \mathrm{bpm}, 95 \%$ CI 1.28,2.50, $\mathrm{p}=0.001$ ). Likewise, patients with a history of coronary heart disease (myocardial infarction, hospital admission for unstable angina or coronary revascularization) who had a faster resting heart rate were at particularly high risk (fully adjusted HR for death 1.23 per $10 \mathrm{bpm}, 95 \%$ CI $1.10,1.37, \mathrm{p}<0.001$ in patients with prior coronary heart disease and fully adjusted HR 1.13 per $10 \mathrm{bpm}, 95 \%$ CI 1.06,1.21, $\mathrm{p}<0.001$ in patients without any known coronary heart disease).

The relationship between baseline heart rate and death is similar regardless of therapy with $\beta$-blockers ( $\mathrm{p}$ for interaction between $\beta$-blocker use and heart rate $=0.57$; figure 3 ). Eight hundred and forty-six patients had current or previous, ECG-confirmed, atrial fibrillation. Of 
these patients, 431 were in atrial fibrillation on the ECG performed at their baseline visit. The relatively small number of patients with current or prior atrial fibrillation limits the power to detect any association between heart rate and outcome in this subgroup. Therefore, although no interaction between atrial fibrillation and heart rate was detected statistically $(\mathrm{p}$ for interaction $=0.62$; figure 3 ), no definitive conclusions can be drawn in this population.

\section{DISCUSSION}

The current data confirm that in patients with type 2 diabetes mellitus a higher resting heart rate is a predictor of all-cause mortality, cardiovascular death and major cardiovascular events. This association remains after adjustment for a variety of other factors that may influence heart rate and impact on outcome in this setting. Indeed, the strength of the relationship is stronger in these adjusted models. It is more evident among diabetic patients who have had a prior vascular event, present in both men and women and is consistent regardless of treatment with $\beta$-blockers.

\section{Comparison with prior studies}

There are few data assessing the prognostic importance of resting heart rate in patients with type 2 diabetes mellitus. Long-term follow up of 302 such patients recruited into the Swiss cohort of the World Health Organisation Multinational Study of Vascular Disease in Diabetes did, however, report a relationship between resting heart rate and both all-cause and cardiovascular mortality.[9] This relationship was maintained after accounting for a variety of potentially confounding factors, though in that population no adjustments were made for the use of $\beta$-blockers, participation in regular exercise or HbA1c. Interestingly, a similar relationship was not observed in patients with type 1 diabetes mellitus.[9]

Recently, the Euro Heart Survey Investigators have assessed the relationship between heart rate and 1 year outcome in 751 diabetic patients and 1,756 non-diabetics, all with stable coronary heart disease.[10] The main finding of this study was that in the diabetic patients a higher resting heart rate was associated with an increased risk of all-cause mortality. This relationship persisted after adjustment for a range of clinical factors though there were only 37 deaths in the diabetic cohort, limiting the ability to accurately correct for potential confounders. Likewise, that study did not account for the type or duration of diabetes, nor any measure of diabetic control or microvascular disease.

In the Euro Heart Survey cohort, there was no significant relationship between resting heart rate and a composite outcome of death, myocardial infarction and stroke.[10] The absence of an association when non-fatal cardiovascular events were considered in addition to all-cause death might imply that the link between higher heart rate and death was driven primarily by non-cardiovascular events. Our data, which address this issue in a much larger cohort followed for considerably longer, suggest that this is not the case. This is consistent with the data in healthy men[11] and in patients with coronary heart disease.[12] It also concurs with the data from Switzerland[9] and with two other small studies which although focussing on electrocardiographically derived QT interval parameters also report an association between resting heart rate and cardiovascular outcome in patients with diabetes.[13, 14] An alternate explanation for the Euro Heart Survey findings is that resting heart rate is more closely 
associated with fatal than non-fatal cardiovascular events. This has been reported in other cohorts[15] but is not evident in the current study.

The nature of any relationship between resting heart rate and cardiovascular events remains to be fully defined. Several studies have reported a linear relationship[16, 17] but a variety of other patterns have also been described, including J-shaped,[18, 19] U-shaped[20] and sigmoid[21] associations. In the current cohort the relationship appears to be predominantly linear but with a suggestion of attenuation at lower heart rates.

\section{Potential mechanisms}

The key question is whether a high resting heart rate is partly causative of an adverse outcome (and if so to what extent) or whether it is merely a marker of other factors that determine prognosis, in particular concomitant disease and/or autonomic dysfunction. Several mechanisms have been proposed whereby a more rapid heart rate might be directly deleterious, all of which apply at least as much to patients with diabetes as to the general population. These include the detrimental effects of a high heart rate on myocardial oxygen demand and supply[1], long-term adverse effects on the vasculature[22-24] and myocardial function [25] and an increased risk of ventricular dysrhythmia[1,2] and sudden cardiac death.[11] In addition to direct effects, an increased heart rate is associated with a variety of factors that may themselves adversely affect prognosis, such as poorer general fitness, obesity, high blood pressure and a less favourable lipid profile,[26, 27] all of which are strongly associated with type 2 diabetes. As far as possible, the current analyses adjust for these potential confounders.

Diabetes may also directly influence resting heart rate. Hyperinsulinemia and elevated blood glucose levels have both been associated with a higher heart rate.[28-30] Furthermore, dysfunction of the autonomic nervous system is a common complication of diabetes, even in the absence of other overt neuropathic complications.[31-33] Damage to cardiovascular autonomic nerve fibres results in sympathetic dominance, with higher resting heart rate, and has been associated with a greatly increased risk of death.[31,34] Evidence of cardiac autonomic neuropathy can be detected early after diagnosis in many patients with diabetes $[31,35]$ and autonomic dysfunction may even pre-date the diagnosis of diabetes itself.[36, 37] In healthy adults recruited in the Atherosclerosis Risk in Communities Study a resting heart rate in the upper quarter was associated with a $60 \%$ increased risk of developing diabetes as compared to those with heart rates in the lowest quarter.[37] This may just reflect an association between heart rate and physical fitness but it underscores the complex interrelationship between diabetes and heart rate.

Randomised studies provide some limited evidence that heart rate plays a direct role in outcome. Beta-blockers reduce mortality after acute myocardial infarction and in patients with heart failure and at least some of the benefits appear to be secondary to a reduction in heart rate.[38, 39] It is, however, difficult to exclude other effects of beta-blockade. In contrast, ivabradine selectively reduces heart rate without other direct effects. In the BEAUTIFUL study ivabradine was compared to placebo in patients with coronary heart disease and left ventricular systolic dysfunction and no overall benefit was demonstrated, though in the pre-specified subgroup with a baseline heart rate $\geq 70 \mathrm{bpm}$ a significant 
reduction in the secondary end-point of myocardial infarction or coronary revascularisation was observed.[40] In the SHIFT study of patients with stable chronic heart failure, ivabradine reduced the primary endpoint, a composite endpoint of cardiovascular death and hospitalization due to heart failure, by $18 \%$ (95\% CI 10\%,25\%),[41] with changes in heart rate appearing to explain most of the observed benefit.[42] Overall, therefore, despite a strong pathophysiological basis, the evidence that heart rate exerts a direct causative role remains limited. Likewise, there is no definitive evidence to support the use of specific ratelimiting medication to improve outcome, except perhaps in patients with stable heart failure.

\section{Strengths and limitations}

The current study is by far the largest to explore the relationship between heart rate and outcome in patients with diabetes to date. The patient population has been drawn from many different countries and is ethnically diverse. It is well characterised and has been carefully followed. Importantly, the number of independently adjudicated outcomes ensures that the study has considerable power and allows statistical adjustment for multiple potentially confounding factors, including several that have not been addressed by prior studies. Nonetheless, it is impossible to fully correct for all potential confounders and, in particular, no specific measures of cardiac autonomic neuropathy are available. Likewise, although we corrected for a prior history of heart failure requiring hospital admission, we were unable to adjust for the presence or severity of any heart failure symptoms at baseline.

\section{Conclusion}

Among patients with diabetes a higher heart rate is associated with increased risk of death and cardiovascular complications. It remains unclear, however, whether a higher heart rate directly mediates the increased risk or whether it is a marker for other characteristics that determine a poor outcome.

\section{Supplementary Material}

Refer to Web version on PubMed Central for supplementary material.

\section{Acknowledgments}

Funding sources: The ADVANCE study was funded by Servier and the National Health and Medical Research Council of Australia (Grant numbers 211086 and 358395). Neither funding body had any input into the analysis or interpretation of the data.

\section{REFERENCES}

[1]. Fox K, Borer JS, Camm AJ, et al. Resting heart rate in cardiovascular disease. J Am Coll Cardiol. 2007; 50:823-830. [PubMed: 17719466]

[2]. Palatini P. Elevated heart rate in cardiovascular diseases: a target for treatment? Prog Cardiovasc Dis. 2009; 52:46-60. [PubMed: 19615493]

[3]. Patel A, MacMahon S, Chalmers J, et al. Effects of a fixed combination of perindopril and indapamide on macrovascular and microvascular outcomes in patients with type 2 diabetes mellitus (the ADVANCE trial): a randomised controlled trial. Lancet. 2007; 370:829-840. [PubMed: 17765963] 
[4]. Patel A, MacMahon S, Chalmers J, et al. Intensive blood glucose control and vascular outcomes in patients with type 2 diabetes. N Engl J Med. 2008; 358:2560-2572. [PubMed: 18539916]

[5]. ADVANCE study group. Study rationale and design of ADVANCE: action in diabetes and vascular disease--preterax and diamicron MR controlled evaluation. Diabetologia. 2001; 44:1118-1120. [PubMed: 11596665]

[6]. Du X, Ninomiya T, de Galan B, et al. Risks of cardiovascular events and effects of routine blood pressure lowering among patients with type 2 diabetes and atrial fibrillation: results of the ADVANCE study. Eur Heart J. 2009; 30:1128-1135. [PubMed: 19282274]

[7]. Woodward, M. Epidemiology: Study Design and Data Analysis. Chapman and Hall/CRC Press; Boca Raton: 2005.

[8]. Lau B, Cole SR, Gange S. Competing Risk Regression Models for Epidemiologic Data. Am J Epidemiol. 2009; 170:244-256. [PubMed: 19494242]

[9]. Stettler C, Bearth A, Allemann S, et al. QTc interval and resting heart rate as long-term predictors of mortality in type 1 and type 2 diabetes mellitus: a 23-year follow-up. Diabetologia. 2007; 50:186-194. [PubMed: 17096116]

[10]. Anselmino M, Ohrvik J, Ryden L. Resting heart rate in patients with stable coronary artery disease and diabetes: a report from the Euro Heart Survey on Diabetes and the Heart. Eur Heart J. 2010; 31:3040-3045. [PubMed: 20935002]

[11]. Jouven X, Empana JP, Schwartz PJ, Desnos M, Courbon D, Ducimetiere P. Heart-rate profile during exercise as a predictor of sudden death. N Engl J Med. 2005; 352:1951-1958. [PubMed: 15888695]

[12]. Diaz A, Bourassa MG, Guertin MC, Tardif JC. Long-term prognostic value of resting heart rate in patients with suspected or proven coronary artery disease. Eur Heart J. 2005; 26:967-974. [PubMed: 15774493]

[13]. Linnemann B, Janka HU. Prolonged QTc interval and elevated heart rate identify the type 2 diabetic patient at high risk for cardiovascular death. The Bremen Diabetes Study. Exp Clin Endocrinol Diabetes. 2003; 111:215-222. [PubMed: 12845560]

[14]. Cardoso CR, Salles GF, Deccache W. Prognostic value of QT interval parameters in type 2 diabetes mellitus: results of a long-term follow-up prospective study. J Diabetes Complications. 2003; 17:169-178. [PubMed: 12810239]

[15]. Poulter NR, Dobson JE, Sever PS, Dahlof B, Wedel H, Campbell NR. Baseline heart rate, antihypertensive treatment, and prevention of cardiovascular outcomes in ASCOT (AngloScandinavian Cardiac Outcomes Trial). J Am Coll Cardiol. 2009; 54:1154-1161. [PubMed: 19761936]

[16]. Mensink GB, Hoffmeister H. The relationship between resting heart rate and all-cause, cardiovascular and cancer mortality. Eur Heart J. 1997; 18:1404-1410. [PubMed: 9458445]

[17]. Seccareccia F, Pannozzo F, Dima F, et al. Heart rate as a predictor of mortality: the MATISS project. Am J Public Health. 2001; 91:1258-1263. [PubMed: 11499115]

[18]. Fujiura Y, Adachi H, Tsuruta M, Jacobs DR Jr. Hirai Y, Imaizumi T. Heart rate and mortality in a Japanese general population: an 18-year follow-up study. J Clin Epidemiol. 2001; 54:495-500. [PubMed: 11337213]

[19]. Kuzuya M, Enoki H, Iwata M, Hasegawa J, Hirakawa Y. J-shaped relationship between resting pulse rate and all-cause mortality in community-dwelling older people with disabilities. $\mathrm{J}$ Am Geriatr Soc. 2008; 56:367-368. [PubMed: 18251825]

[20]. Dyer AR, Persky V, Stamler J, et al. Heart rate as a prognostic factor for coronary heart disease and mortality: findings in three Chicago epidemiologic studies. Am J Epidemiol. 1980; 112:736749. [PubMed: 7457467]

[21]. Palatini P, Casiglia E, Julius S, Pessina AC. High heart rate: a risk factor for cardiovascular death in elderly men. Arch Intern Med. 1999; 159:585-592. [PubMed: 10090115]

[22]. Sa Cunha R, Pannier B, Benetos A, et al. Association between high heart rate and high arterial rigidity in normotensive and hypertensive subjects. J Hypertens. 1997; 15:1423-1430. [PubMed: 9431848]

[23]. Heidland UE, Strauer BE. Left ventricular muscle mass and elevated heart rate are associated with coronary plaque disruption. Circulation. 2001; 104:1477-1482. [PubMed: 11571239] 
[24]. Huikuri HV, Jokinen V, Syvanne M, et al. Heart rate variability and progression of coronary atherosclerosis. Arterioscler Thromb Vasc Biol. 1999; 19:1979-1985. [PubMed: 10446081]

[25]. Spinale FG, Hendrick DA, Crawford FA, Smith AC, Hamada Y, Carabello BA. Chronic supraventricular tachycardia causes ventricular dysfunction and subendocardial injury in swine. Am J Physiol. 1990; 259:H218-229. [PubMed: 2375409]

[26]. Jurca R, Jackson AS, LaMonte MJ, et al. Assessing cardiorespiratory fitness without performing exercise testing. Am J Prev Med. 2005; 29:185-193. [PubMed: 16168867]

[27]. Palatini P, Julius S. The physiological determinants and risk correlations of elevated heart rate. Am J Hypertens. 1999; 12:3S-8S. [PubMed: 10077413]

[28]. Yeap BB, Russo A, Fraser RJ, Wittert GA, Horowitz M. Hyperglycemia affects cardiovascular autonomic nerve function in normal subjects. Diabetes Care. 1996; 19:880-882. [PubMed: 8842608]

[29]. Marfella R, Nappo F, De Angelis L, Siniscalchi M, Rossi F, Giugliano D. The effect of acute hyperglycaemia on QTc duration in healthy man. Diabetologia. 2000; 43:571-575. [PubMed: 10855531]

[30]. Anderson EA, Hoffman RP, Balon TW, Sinkey CA, Mark AL. Hyperinsulinemia produces both sympathetic neural activation and vasodilation in normal humans. J Clin Invest. 1991; 87:22462252. [PubMed: 2040704]

[31]. Vinik AI, Maser RE, Mitchell BD, Freeman R. Diabetic autonomic neuropathy. Diabetes Care. 2003; 26:1553-1579. [PubMed: 12716821]

[32]. Ziegler D, Dannehl K, Muhlen H, Spuler M, Gries FA. Prevalence of cardiovascular autonomic dysfunction assessed by spectral analysis, vector analysis, and standard tests of heart rate variation and blood pressure responses at various stages of diabetic neuropathy. Diabet Med. 1992; 9:806-814. [PubMed: 1473320]

[33]. Ziegler D, Gries FA, Spuler M, Lessmann F. The epidemiology of diabetic neuropathy. Diabetic Cardiovascular Autonomic Neuropathy Multicenter Study Group. J Diabetes Complications. 1992; 6:49-57. [PubMed: 1562759]

[34]. Maser RE, Mitchell BD, Vinik AI, Freeman R. The association between cardiovascular autonomic neuropathy and mortality in individuals with diabetes: a meta-analysis. Diabetes Care. 2003; 26:1895-1901. [PubMed: 12766130]

[35]. Ziegler D, Dannehl K, Volksw D, Muhlen H, Spuler M, Gries FA. Prevalence of cardiovascular autonomic dysfunction assessed by spectral analysis and standard tests of heart-rate variation in newly diagnosed IDDM patients. Diabetes Care. 1992; 15:908-911. [PubMed: 1516513]

[36]. Carnethon MR, Jacobs DR Jr. Sidney S, Liu K. Influence of autonomic nervous system dysfunction on the development of type 2 diabetes: the CARDIA study. Diabetes Care. 2003; 26:3035-3041. [PubMed: 14578236]

[37]. Carnethon MR, Golden SH, Folsom AR, Haskell W, Liao D. Prospective investigation of autonomic nervous system function and the development of type 2 diabetes: the Atherosclerosis Risk In Communities study, 1987-1998. Circulation. 2003; 107:2190-2195. [PubMed: 12695289]

[38]. Kjekshus JK. Importance of heart rate in determining beta-blocker efficacy in acute and longterm acute myocardial infarction intervention trials. Am J Cardiol. 1986; 57:43F-49F.

[39]. Lechat P, Escolano S, Golmard JL, et al. Prognostic value of bisoprolol-induced hemodynamic effects in heart failure during the Cardiac Insufficiency BIsoprolol Study (CIBIS). Circulation. 1997; 96:2197-2205. [PubMed: 9337190]

[40]. Fox K, Ford I, Steg PG, Tendera M, Ferrari R. Ivabradine for patients with stable coronary artery disease and left-ventricular systolic dysfunction (BEAUTIFUL): a randomised, double-blind, placebo-controlled trial. Lancet. 2008; 372:807-816. [PubMed: 18757088]

[41]. Swedberg K, Komajda M, Bohm M, et al. Ivabradine and outcomes in chronic heart failure (SHIFT): a randomised placebo-controlled study. Lancet. 2010; 376:875-885. [PubMed: 20801500]

[42]. Bohm M, Swedberg K, Komajda M, et al. Heart rate as a risk factor in chronic heart failure (SHIFT): the association between heart rate and outcomes in a randomised placebo-controlled trial. Lancet. 2010; 376:886-894. [PubMed: 20801495] 
a

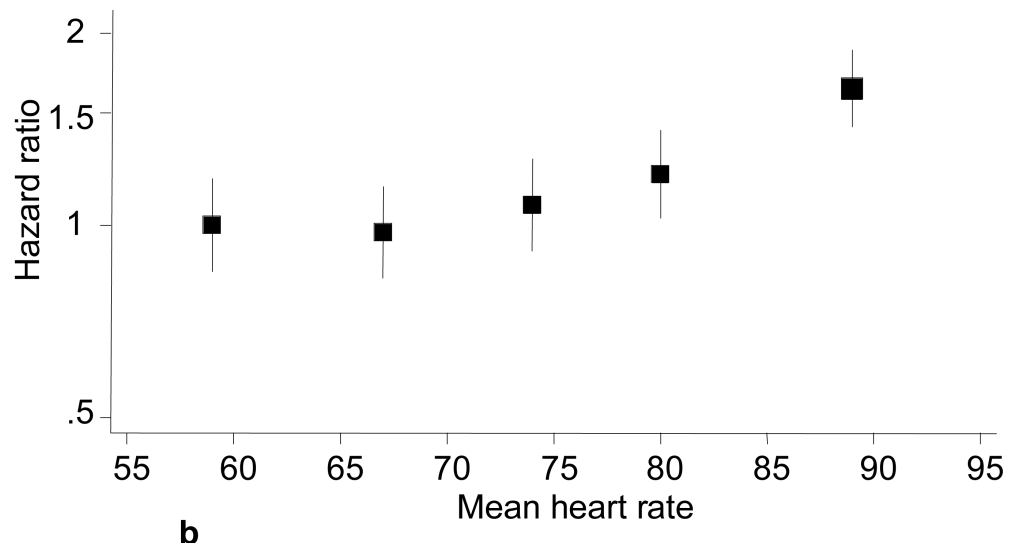

b

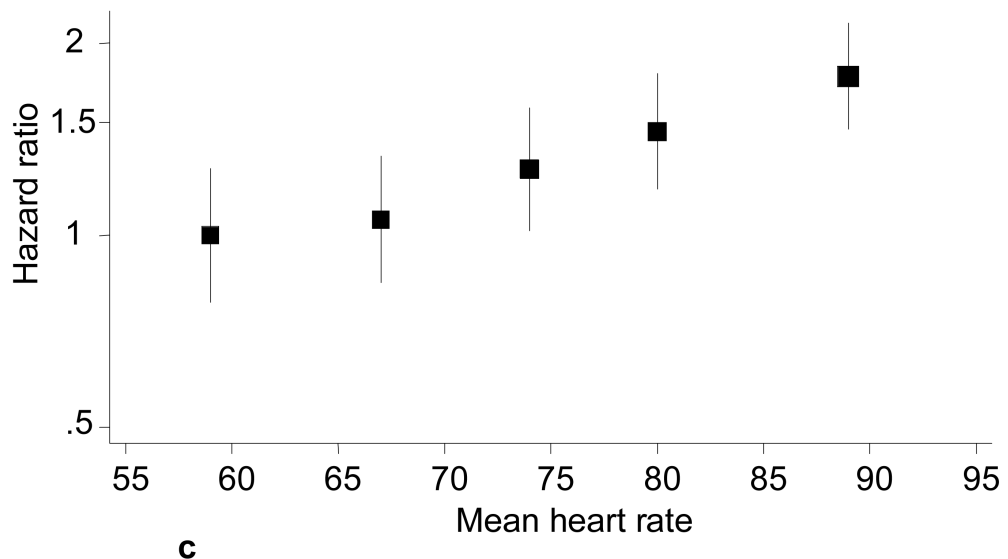

C

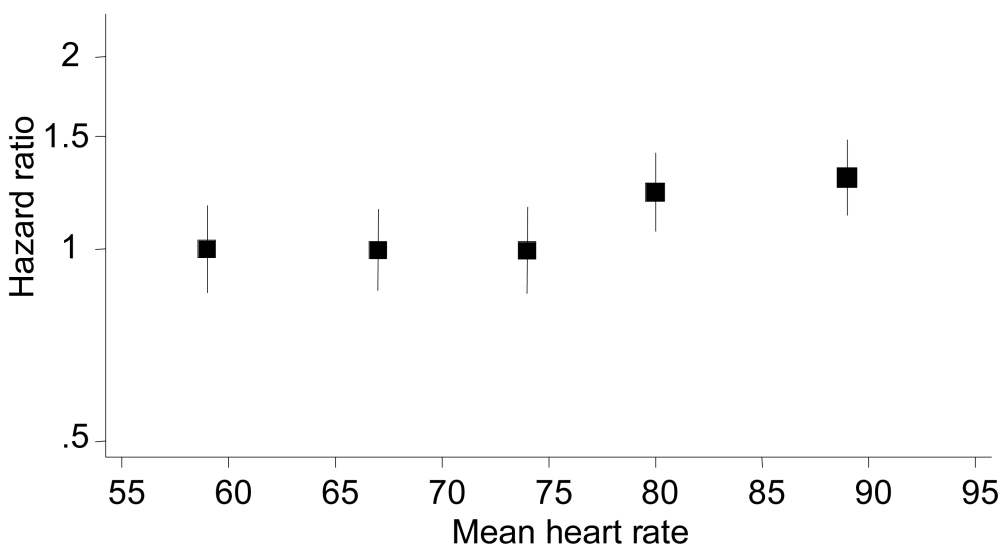

Figure 1. Baseline resting heart rate (by fifths) and the risk of death from any cause, cardiovascular death and major cardiovascular events

Figure 1a Death from any cause $(\mathrm{p}<0.001$ for trend)

Figure 1b Cardiovascular death $(\mathrm{p}<0.001$ for trend)

Figure 1c Major cardiovascular events $(\mathrm{p}=0.002$ for trend)

Hazard adjusted for age, sex, ADVANCE study blood pressure treatment arm, ADVANCE study glycaemic control arm, body mass index, duration of diabetes, HbA1c, urinary albumin creatinine ratio, estimated glomerular filtration rate, systolic blood pressure, diastolic blood pressure, history of hospitalisation for heart failure, participation in moderate 
and/or vigorous exercise for $>15$ minutes at least once weekly, total cholesterol, triacylglycerol level, atrial fibrillation, treatment with calcium channel blockers and treatment with $\beta$-blockers. 
A. Hazard ratio for HR per $10 \mathrm{bpm}$

\section{Death from any cause}

No prior macrovascular disease

Prior macrovascular disease

Cardiovascular death

No prior macrovascular disease

Prior macrovascular disease

Major cardiovascular events

No prior macrovascular disease

Prior macrovascular disease

B. Hazard ratio for $5^{\text {th }} v 1^{\text {st }}$ fifth

Death from any cause

No prior macrovascular disease

Prior macrovascular disease

\section{Cardiovascular death}

No prior macrovascular disease

Prior macrovascular disease

Major cardiovascular events

No prior macrovascular disease

Prior macrovascular disease

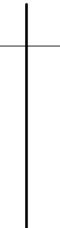

$\div$

$1.14(1.05,1.23) \quad 0.001$

$1.17(1.08,1.28) \quad<0.001$

$1.13(1.01,1.27) \quad 0.03$

$1.20(1.08,1.34) \quad 0.001$

$1.07(0.99,1.15)$

0.10

$1.10(1.02,1.19)$

0.01

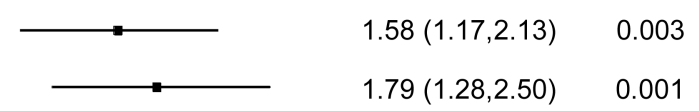

$1.73(1.10,2.75) \quad 0.02$

$1.81(1.21,2.88) \quad 0.005$

$1.34(0.99,1.81)$

0.06

$1.35(1.00,1.83)$

0.05

Figure 2. Baseline resting heart rate and the risk of death from any cause, cardiovascular death and major cardiovascular events stratified by a history of prior macrovascular complications Macrovascular disease: Myocardial infarction, stroke, hospital admission for a transient ischemic attack, hospital admission for unstable angina, coronary revascularization, peripheral revascularization or amputation secondary to peripheral vascular disease. Hazard adjusted for age, sex, ADVANCE study blood pressure treatment arm, ADVANCE study glycaemic control arm, body mass index, duration of diabetes, HbA1c, urinary albumin creatinine ratio, estimated glomerular filtration rate, systolic blood pressure, diastolic blood pressure, history of hospitalisation for heart failure, participation in moderate and/or vigorous exercise for > 15 minutes at least once weekly, total cholesterol, 
triacylglycerol level, atrial fibrillation, treatment with calcium channel blockers and treatment with $\beta$-blockers. 


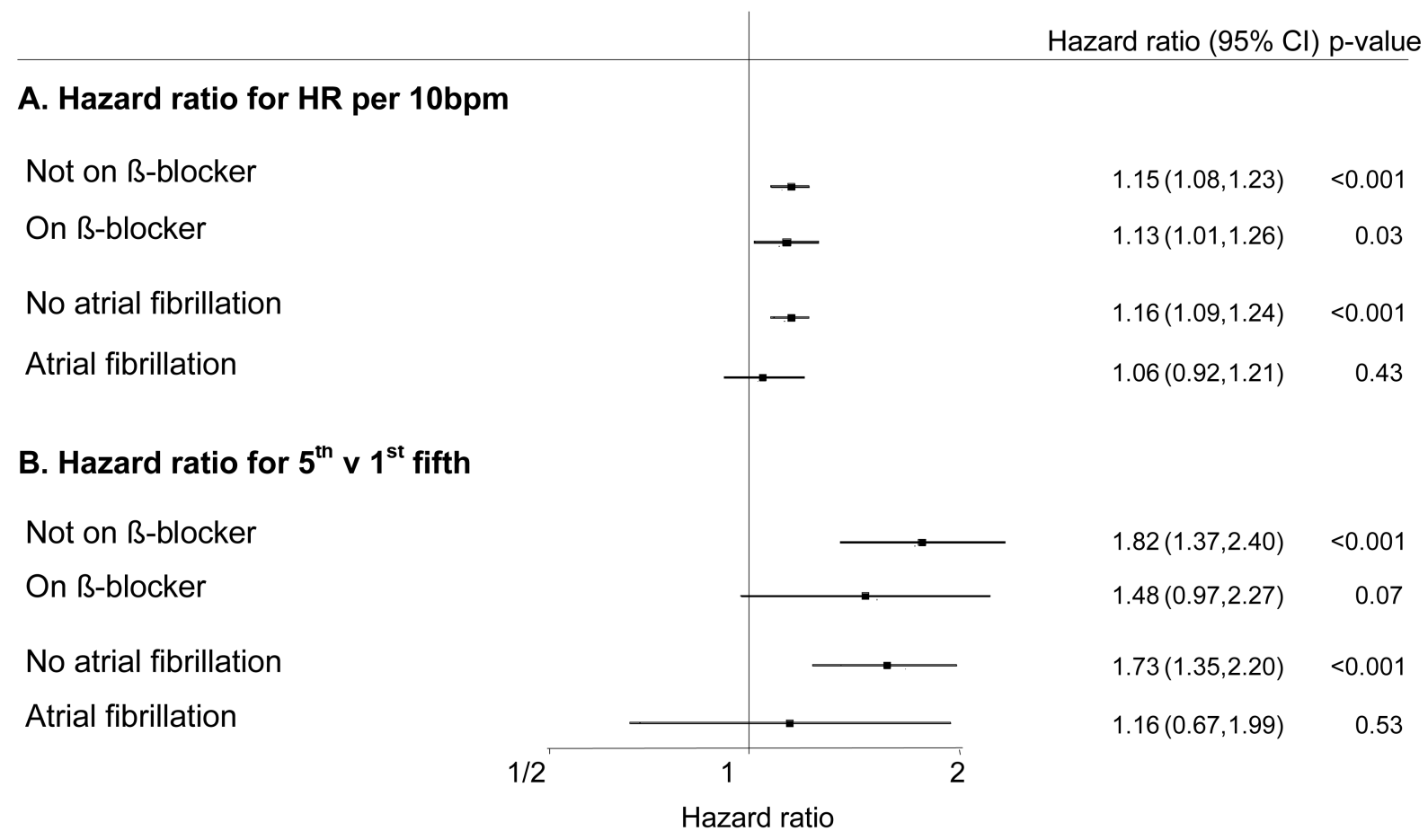

Figure 3. Baseline resting heart rate and the risk of death from any cause stratified by treatment with $\beta$-blockers and by atrial fibrillation

Hazard adjusted for age, sex, ADVANCE study blood pressure treatment arm, ADVANCE study glycaemic control arm, body mass index, duration of diabetes, HbA1c, urinary albumin creatinine ratio, estimated glomerular filtration rate, systolic blood pressure, diastolic blood pressure, history of hospitalisation for heart failure, participation in moderate and/or vigorous exercise for $>15$ minutes at least once weekly, total cholesterol, triacylglycerol level, atrial fibrillation (only in analysis stratified by treatment with $\beta$ blockers), treatment with calcium channel blockers and treatment with $\beta$-blockers (only in analysis stratified by history of atrial fibrillation). 


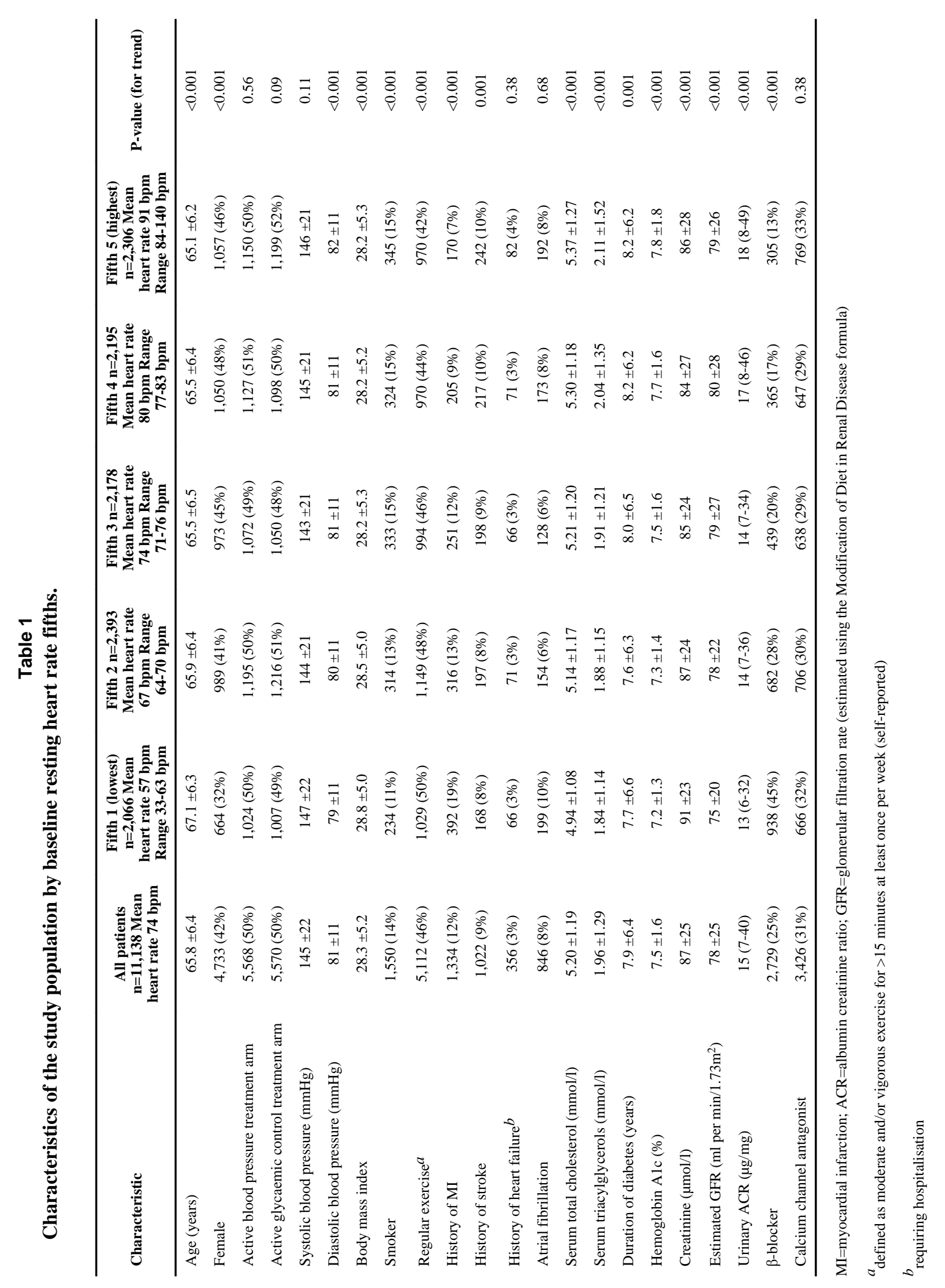

Diabetologia. Author manuscript; available in PMC 2014 September 22. 